\title{
3D Video Conversion Method Based on the Depth of the Image Description
}

\author{
Huiyu Nie \\ BAICHENG NORMAL UNIVERSITY, Baicheng, 137000, China \\ 3174296251@qq.com
}

Keywords: Depth image rendering, 3D video, Conversion method

\begin{abstract}
In this paper, the method of "depth map" is used to convert 2D video to 3D video, and the method of depth map is studied. A method of creating a red and Blue 3D image based on depth image rendering (DIBR) is proposed for the problem of hole in the left view. According to the parallax information of red color offset left view, for the void phenomenon appeared in the primary migration image. Through smooth depth map processing and improved hole filling technology on the left view appears holes have been effective to fill and perfect, reducing the hole filling in computational complexity, forming a two-dimensional video sequence into the effective method of 3D video.
\end{abstract}

\section{Introduction}

With the development of digital video technology, digital video is gradually transition from two-dimensional display to three-dimensional display. At present the development of 3D video met the bottleneck of the lack of sources, stereoscopic film from shooting to issue need specific technology, production costs are high and the cycle is longer. How to convert the existing 2D video to 3D video, it becomes an important research area in 3D technology. In essence, the final effect of the two kinds of technology is the same, it is produced by the traditional 2D video has a certain disparity image pairs, but DIBR technology is mainly through the generation of depth map of the way to transform the disparity map, and virtual viewpoint rendering [1].

\section{D image conversion technology based on DIBR}

The main function of DIBR technology is to synthesize a new view (target image) by using the reference image and its corresponding depth information. The traditional DIBR system mainly consists of three parts: deep image preprocessing, 3D image transform and empty filling [2]. View synthesis technology based on 3D is the key technology in DIBR synthesis. The traditional stereoscopic video needs to transmit the left and right channels. The DIBR can omit the complex production process, which greatly reduces the bandwidth and the production cycle, and the use of 2D/3D conversion technology to facilitate the support of a variety of three-dimensional video.

DIBR is the process of using the reference map and the depth information of the corresponding pixel point to synthesize the virtual viewpoint in the scene [3], the detailed steps are as follows.

Depth image preprocessing.

In the depth map, the depth value changes in the region (usually at the edge of the moving object), which may produce larger voids after 3D image changes. The depth map can reduce the number of holes or reduce the size of holes. So this paper is selected from the Gauss filter to deal with the depth map, the next chapter will prove that the depth of the hole is reduced, but the image quality is not obvious. The image quality is not obvious, and the hole of the target is reduced.

\section{D image transform.}

The 3D image transform is used to map the pixels in the reference image to the target image in the depth map. It is possible to map a few pixels in the reference image to the same point of the target image when the 3D image transform is used in the target image. Cavity is one of the most typical problems, especially when the position deviation of the target image and the reference image is relatively large, it is easy to produce a large hole. 


\section{Void Filling.}

How to fill in the hole has become a key point in the DIBR technology, this paper will use one kind of hole filling technology based on the original image and depth information to achieve this goal. For video conversion, each frame of the video can be extracted, followed by the DIBR system in order to obtain a set of images, this group of images in accordance with the speed of 24 frames per second made of red blue 3D video. Since the core of video conversion is image conversion, the process of image transformation is introduced in detail, and the flow chart of 2D video conversion is introduced, and the $3 \mathrm{D}$ image sequence is obtained by experiments using 2D video sequence.

\section{Dimensional Video Conversion Method}

\section{Principles of conversion method.}

In this paper, a new method is developed for a two dimensional video conversion process, based on the core technology of 3D video converting.

By the disparity map and the original video frame is red color component images, created by doing basic color deviation left view, due to the presence of cavitation generated left view, the hole filling technique on the left view, hole filling, to eliminate the empty hole phenomenon in the left view. Finally, the elimination of the left view of the cavitation and created by the green color component of the original image right view, via view synthesis technology create three-dimensional images of the red and blue.

\section{Experimental result.}

Hall video and walk video conversion experiments, the experimental results are shown in figure 1.

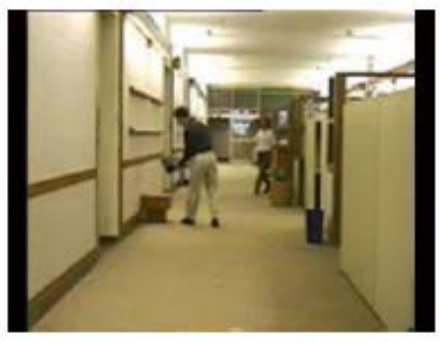

Frame 93

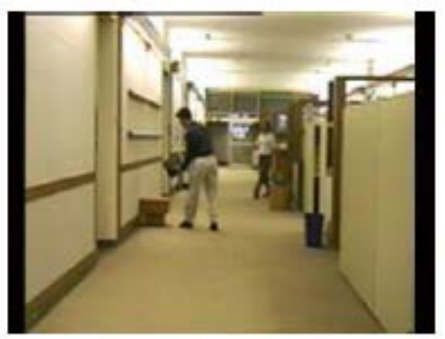

Frame 96

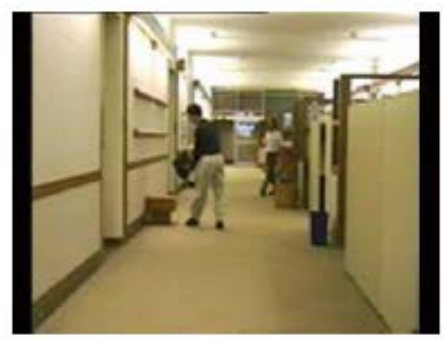

Frame 94

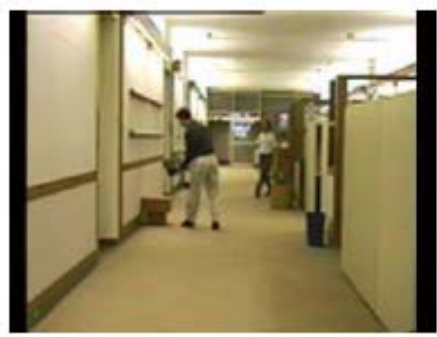

Frame 97

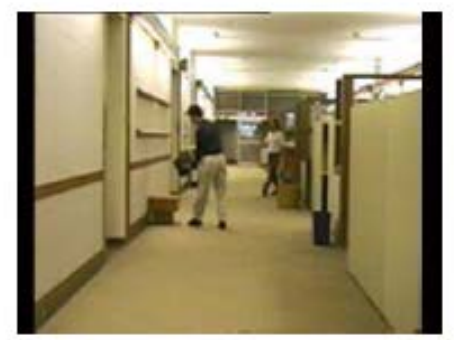

Frame 95

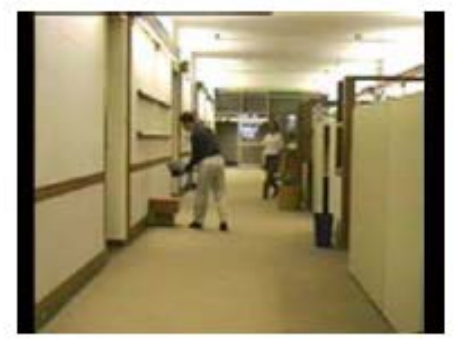

Frame98

(a) Original hall video sequence 


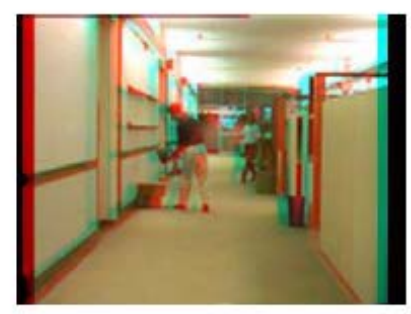

Frame 93

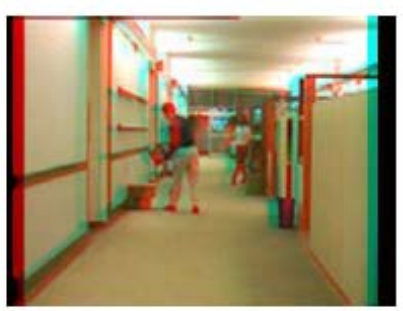

Frame 96

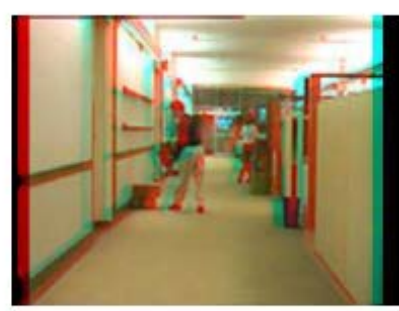

Frame 94

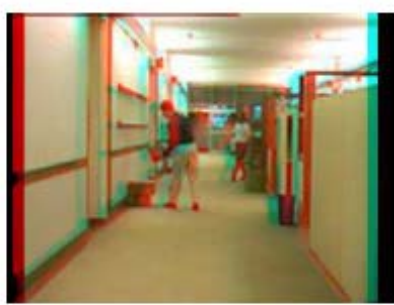

Frame 97

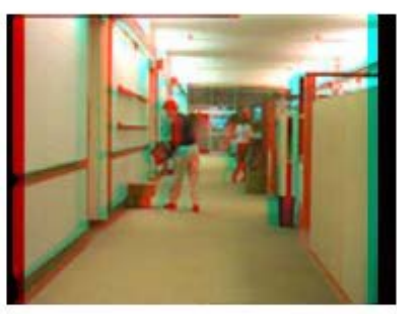

Frame 95

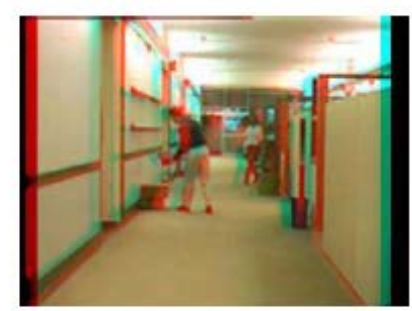

Frame98

(b) Video sequence after the experiment

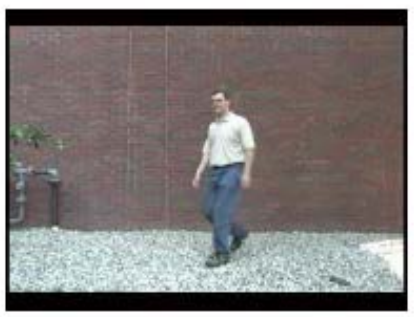

Frame 66

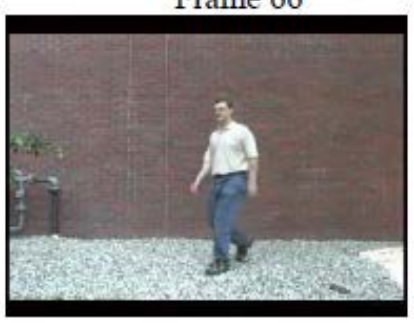

Frame 69

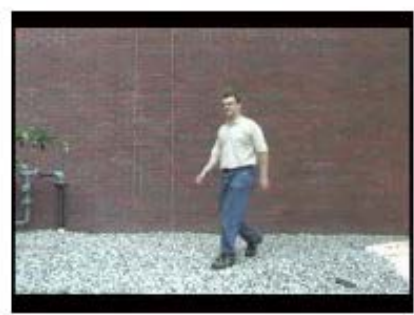

Frame 67

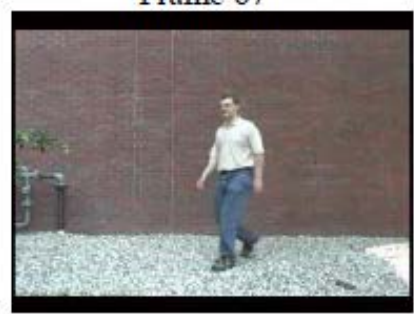

Frame 70

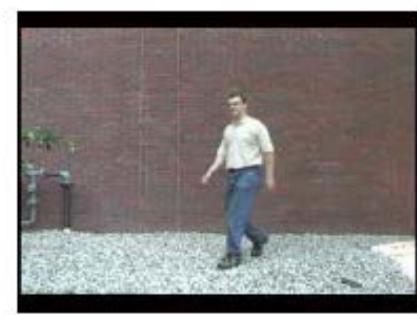

Frame 68

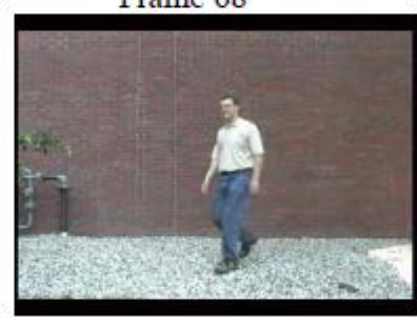

Frame71

(c) Original video framesfor Walk

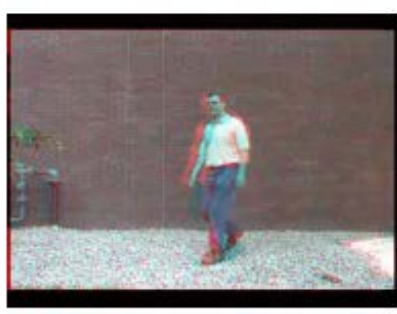

Frame 66

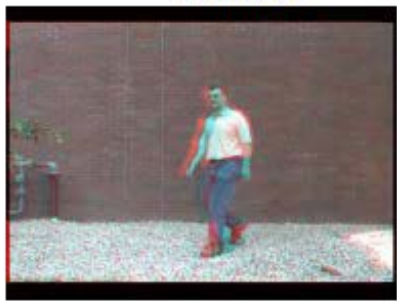

Frame 69

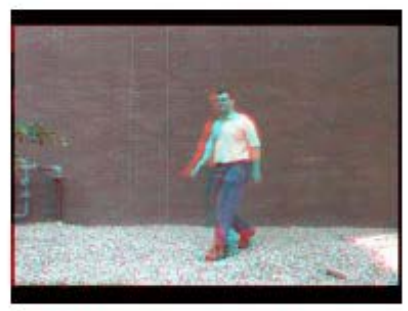

Frame 67

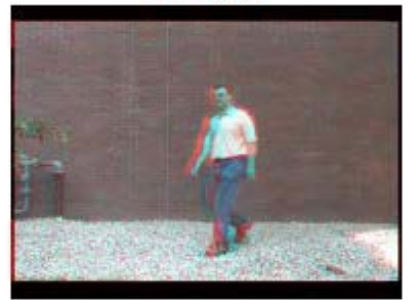

Frame 70

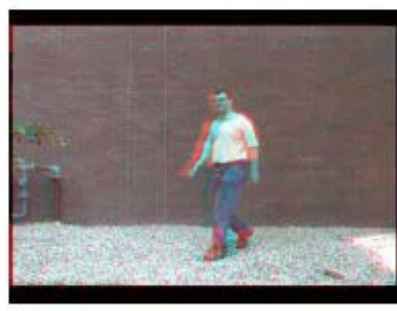

Frame 68

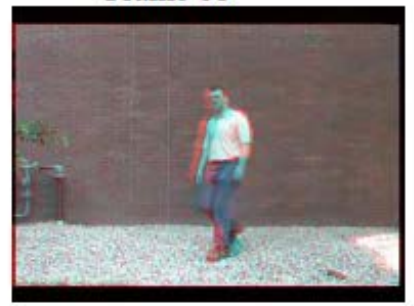

Frame71

(d) 3D video frames

Fig. 1 3D video sequences 
By wearing a red blue 3D glasses to watch, a strong three-dimensional effect, this paper shows the effectiveness of the two-dimensional video conversion method, and in real-time, the program uses the software is running, the walk video sequences of 136 frames are converted into red and blue 3D sequences, which takes two seconds, and the real-time performance is still to be improved, which is the next step in this paper.

\section{Summary}

This paper introduces the principle of image rendering technology (DIBR) based on depth map (DIBR), and the three parts of 3D conversion system: depth map preprocessing, 3D image conversion, void filling. In the end, the experimental results of the process of generating the red and blue 3D images by using the DIBR system are presented.

\section{References}

[1] Zhanwei Liu,Ping An,Suxing Liu.Any view point to draw based on the DIBR and image fusion[J]. Journal of Image and Graphics, 2007, 12(10): 1696-1700.

[2] Kai Luo,Dongxiao Li,Yamei Feng.Any view point to draw based on the DIBR and image repair [J]. Journal of Image and Graphics,2010,(3): 443-449.

[3] Ran Liu, Qingsheng Zhu,Lin Yi.View synthesis Based on image projection [J]. Computer application , 2010,(4): 898-901.

[4] Fehn C. A 3DTV approach using depth-image based rendering(DIBR)[C]. Proceedings of Visualization, Imaging, and Image Processing, 2003: 482-487.

[5] Chen,W-Y.,Y.-L.Chang,S.-F.Lin,L.-F. Ding,L.-G. Chen. Efficient depth image based rendering with edge dependent depth filter and interpolation[M]. Proceedings of IEEE International Conference,Multimedia andExpo.2005. Amsterdam,Netherland: Institute of Electrical and Electronics Engineers Computer Society:1314-1317. 